\title{
On-axis interferometric detection using a binary joint transform correlator
}

\author{
Ignasi Labastida *, Arturo Carnicer, Ignacio Juvells, Santiago Vallmitjana \\ Universitat de Barcelona, Departament de Física Aplicada i Òptica, Av. Diagonal 647, E08028 Barcelona, Spain
}

\begin{abstract}
Recently, we proposed an approach to obtain a single on-axis correlation with use of a joint transform correlator. That method required a displaying device with a determinate configuration, however, in this work we introduce a modification to be used with a modulator without a full $2 \pi$ phase modulation. This variation is based on a binary joint power spectrum and it requires an interferometric process to obtain a single detection peak. We present a setup to carry out the whole process optically and we show experimental results that validate this new approach.
\end{abstract}

Keywords: Pattern recognition; Optical correlation; Joint transform correlator; Liquid crystal displays

\section{Introduction}

The joint transform correlator (JTC) [1,2] has been widely used as an architecture to implement correlation in optical pattern recognition field. Although many improvements have been proposed to obtain real-time detection and to increase the discrimination capability, for instance [3,4], there is still a remaining issue in JTCs: to obtain an isolate output correlation, due to the zero-order term and the two symmetrically non centered correlation terms that appear at the output plane. Different approaches have

\footnotetext{
* Corresponding author. Tel: +34-934021203; fax: +34934021142; e-mail: nasi@optica.ub.es
}

been proposed to deal with this problem: to focus each diffracted term on a different plane $[5,6]$, to use phase-shifting methods to get a non-zero-order spectrum $[7,8]$ or to remove the zero-order diffraction term [9].

Recently we proposed an approach not to have only a non-zero-order joint transform correlation, but also obtain a single centered detection term at the output plane using a JTC [10]. The method was based on a four-level power spectrum that required a special configuration to be displayed on a liquidcrystal panel. In the present work, we introduce a modification of our previous approach using now a binary power spectrum. The requirements to display this binary spectrum on a modulator are less restrictive than the needed for the four-level, allowing the 
use of different configurations. This method can be useful for liquid-crystal displays (LCD) that can not reach a $2 \pi$ phase modulation, as many of the ones removed from commercial videoprojectors, widely used in optical setups. In this paper we propose a suitable setup based on an interferometric architecture to implement our method and we show some experimental results we have obtained.

\section{On-axis joint transform correlation}

The method that we proposed in our previous work [10] was based on the use of a four-level joint power spectrum (JPS). Our goal was to obtain a single detection peak at the output plane of a JTC:

$c(x, y) \propto \delta\left(x-x_{\mathrm{r}}, y-y_{\mathrm{r}}\right)$,

where $\left(x_{\mathrm{r}}, y_{\mathrm{r}}\right)$ was the position of the reference into the scene. For that purpose we suggested to compute the following complex transmittance:

$\exp \left(\mathrm{i}\left(\phi_{\mathrm{R}}(u, v)-\phi_{\mathrm{S}}(u, v)\right)\right)$,

where $\phi_{\mathrm{R}}(u, v)$ and $\phi_{\mathrm{S}}(u, v)$ were the phase of the Fourier transform of the reference and the scene, respectively. The Fourier transform of the aforementioned complex transmittance (relation (2)) gives the desired single centered detection peak (relation (1)). To achieve relation (2), we defined the following intensity distribution:

$I_{\mathrm{q}}(u, v)$

$$
=\left\{\begin{array}{rlll}
1+\mathrm{i} & \text { if } I_{\mathrm{C}}(u, v) \geq I_{\mathrm{T}}(u, v) & \text { AND } & I_{\mathrm{S}}(u, v) \geq I_{\mathrm{T}}(u, v) \\
-1+\mathrm{i} & \text { if } I_{\mathrm{C}}(u, v)<I_{\mathrm{T}}(u, v) & \text { AND } & I_{\mathrm{S}}(u, v) \geq I_{\mathrm{T}}(u, v) \\
1-\mathrm{i} & \text { if } I_{\mathrm{C}}(u, v) \geq I_{\mathrm{T}}(u, v) & \text { AND } & I_{\mathrm{S}}(u, v)<I_{\mathrm{T}}(u, v) \\
-1-\mathrm{i} & \text { if } I_{\mathrm{C}}(u, v)<I_{\mathrm{T}}(u, v) & \text { AND } & I_{\mathrm{S}}(u, v)<I_{\mathrm{T}}(u, v)
\end{array}\right.
$$

where

$$
\begin{aligned}
I_{\mathrm{C}}(u, v)= & \left|F_{\mathrm{R}}(u, v)\right|^{2}+\left|F_{\mathrm{S}}(u, v)\right|^{2} \\
& +2\left|F_{\mathrm{R}}(u, v)\right|\left|F_{\mathrm{S}}(u, v)\right| \cos \left(\phi_{\mathrm{R}}(u, v)\right. \\
& \left.-\phi_{\mathrm{S}}(u, v)\right),
\end{aligned}
$$

$$
\begin{aligned}
I_{\mathrm{S}}(u, v)= & \left|F_{\mathrm{R}}(u, v)\right|^{2}+\left|F_{\mathrm{S}}(u, v)\right|^{2} \\
& +2\left|F_{\mathrm{R}}(u, v)\right|\left|F_{\mathrm{S}}(u, v)\right| \sin \left(\phi_{\mathrm{R}}(u, v)\right. \\
& \left.-\phi_{\mathrm{S}}(u, v)\right)
\end{aligned}
$$

and $I_{\mathrm{T}}(u, v)$ was a threshold function. Note that $F_{\mathrm{R}}(u, v)$ and $F_{\mathrm{S}}(u, v)$ are the complex Fourier transforms of the reference and the scene images, respectively.

When $I_{\mathrm{T}}(u, v)=\left|F_{\mathrm{R}}(u, v)\right|^{2}+\left|F_{\mathrm{S}}(u, v)\right|^{2}$, the distribution $I_{\mathrm{q}}(u, v)$ could be written as [11]:

$$
\begin{aligned}
I_{\mathrm{q}}(u, v) \approx & \cos \left(\phi_{\mathrm{R}}(u, v)-\phi_{\mathrm{S}}(u, v)\right) \\
& +\operatorname{isin}\left(\phi_{\mathrm{R}}(u, v)-\phi_{\mathrm{S}}(u, v)\right) \\
= & \exp \left(\mathrm{i}\left(\phi_{\mathrm{R}}(u, v)-\phi_{\mathrm{S}}(u, v)\right)\right) .
\end{aligned}
$$

To perform all this process optically we needed four grey levels that had the same transmittance and with relative phase shift of $\pi / 2$, as pointed out in Eq. (3).

Now, we propose to reduce these four grey levels to only two, that also must have the same transmittance but with relative phase shift of $\pi$. This procedure is similar to the binarization of the JPS [12], but we use a sine distribution (Eq. (5)) instead of a cosine one. Another difference is that our method gives a single centered detection peak by displaying the scene and the reference superimposed on the input modulator. Therefore, both the input and the correlation plane images are on-axis and the space bandwidth product of the CCD camera can be optimized with respect to the acquisition of the whole output plane in a conventional JTC.

To obtain the sine distribution of Eq. (5), we introduce the scene and the reference centered at the input plane with a phase shift of $\pi / 2$ between them,

$f_{\mathrm{R}}(x, y)+f_{\mathrm{S}}(x, y) \exp (\mathrm{i} \pi / 2)$.

It is possible to have a phase shift between two images using the modulation properties of the LCDs, which are described by its operating curves, as we will further explain in Section 3. 
We define a binary function $I_{\mathrm{BS}}$ :

$I_{\mathrm{BS}}(u, v)=\left\{\begin{aligned} \mathrm{i} & \text { if } I_{\mathrm{S}}(u, v) \geq I_{\mathrm{T}}(u, v) \\ -\mathrm{i} & \text { if } I_{\mathrm{S}}(u, v)<I_{\mathrm{T}}(u, v)\end{aligned}\right.$

If we use the following threshold function:

$I_{\mathrm{T}}(u, v)=\left|F_{\mathrm{R}}(u, v)\right|^{2}+\left|F_{\mathrm{S}}(u, v)\right|^{2}$

the distribution $I_{\mathrm{BS}}(u, v)$ can be written as:

$I_{\mathrm{BS}}(u, v) \approx \operatorname{isin}\left(\phi_{\mathrm{R}}(u, v)-\phi_{\mathrm{S}}(u, v)\right)$.

The threshold function (Eq. (9)) can be obtained by evaluation of the intensity of the Fourier transform of the scene and the reference separately.

Then, using $I_{\mathrm{BS}}$, with the aforementioned threshold function, we get at the correlation plane:

$c(x, y) \propto \mathrm{i}\left(\delta\left(x-x_{\mathrm{r}}, y-y_{\mathrm{r}}\right)-\delta\left(x+x_{\mathrm{r}}, y+y_{\mathrm{r}}\right)\right)$,

when the reference is included into the scene at the position $\left(x_{\mathrm{r}}, y_{\mathrm{r}}\right)$.
Note that this amplitude distribution will lead us to capture two indistinguishable intensity detection peaks when we register them by means of a CCD camera. Therefore, at the output plane we have two centered peaks without the usual diffraction spot at the center. Now, we have to remove one of these two peaks to get a single term.

Before the correlation plane is captured by a CCD camera we can add a plane wave that will allow, in the ideal case, the elimination of one of the terms of Eq. (11) due to negative interference. When the amplitude of this plane wave is not exactly the same as the one of the terms, the duplicated correlation peak is just reduced. By binarizing the final output plane using a high enough threshold, the resulting background and the possible remaining useless peak can be removed.

\section{Optical setup}

To perform the whole process optically, we need an interferometric setup therefore we propose the architecture sketched in Fig. 1, that is based on a

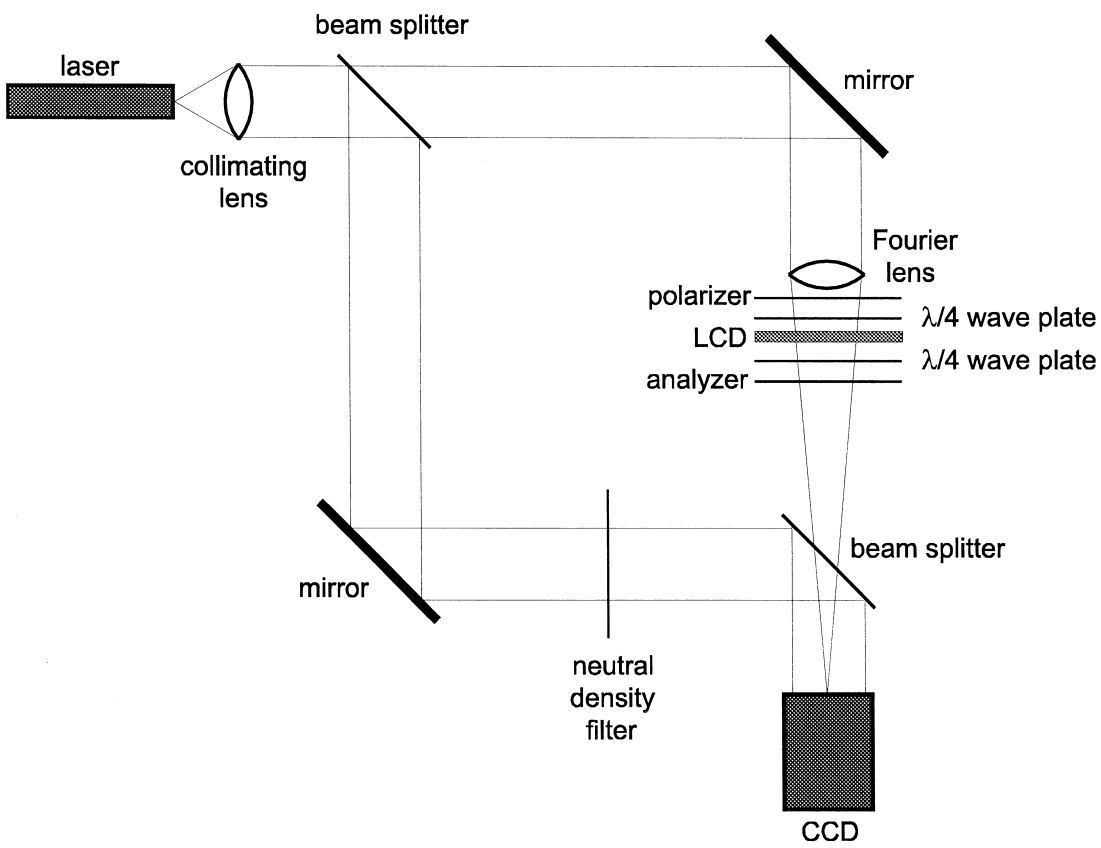

Fig. 1. Optical setup. Polarizers and wave plates are needed to obtain the desired LCD configurations 
Mach-Zehnder interferometer. To obtain the required power spectra we only use one of the arms of the setup, while both arms are used to achieve the final correlation.

In our setup, the images are displayed on an LCD that modulates both the phase and amplitude of the transmitted light, depending on the applied voltage and the polarization of light. This is controlled by the output signal of a VGA video card, that transforms different grey levels of images on a computer in electrical signals. It is then necessary to obtain the operating curves of the panel, which give the relationship between the grey level of the input image and the amplitude and phase modulation. Depending on the orientation of the polarizer and analyzer placed before and after the modulator and on the values of some potentiometer controls available on the videoprojector (brightness and contrast), different kinds of response are obtained.

First, we have to display the reference and the scene images (relation (7)) at the input plane. For that purpose, we could require two LCDs. Both of them should only modulate the amplitude but their operating curves should give a phase shift of $\pi / 2$ between them. In this way, after a Fourier transform, we would obtain $I_{\mathrm{S}}$ (Eq. (5)) by means of the CCD

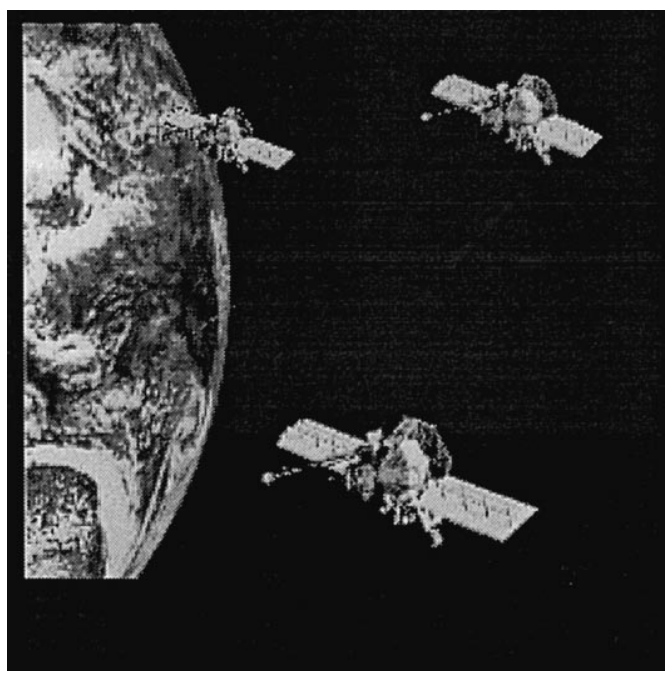

Fig. 2. Image used as scene.

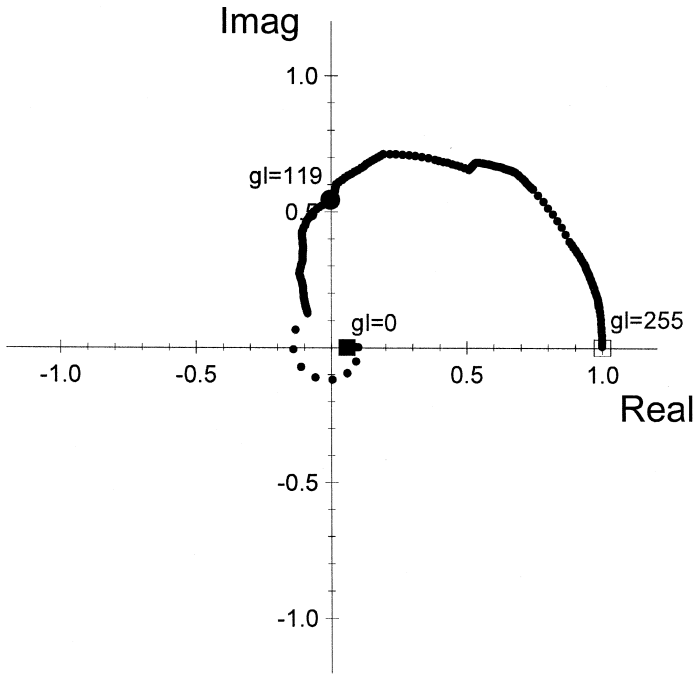

Fig. 3. High-contrast configuration.

camera, so we could compute $I_{\mathrm{BS}}$ (Eq. (8)). As we pointed out in our previous work [10], we can use a single panel with a high-contrast (HC) configuration and binary input images [8].

To perform the second Fourier transform and get the desired centered correlation plane, we need a

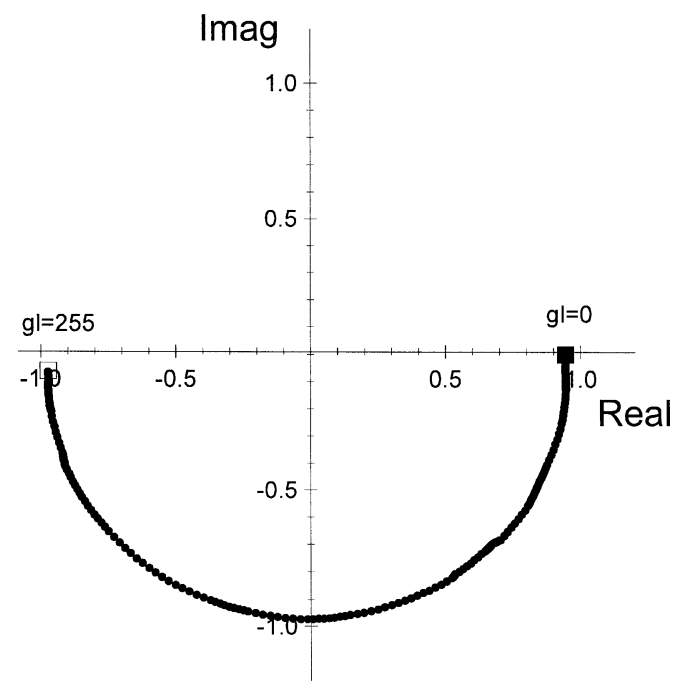

Fig. 4. $0-\pi$ phase-only configuration. 

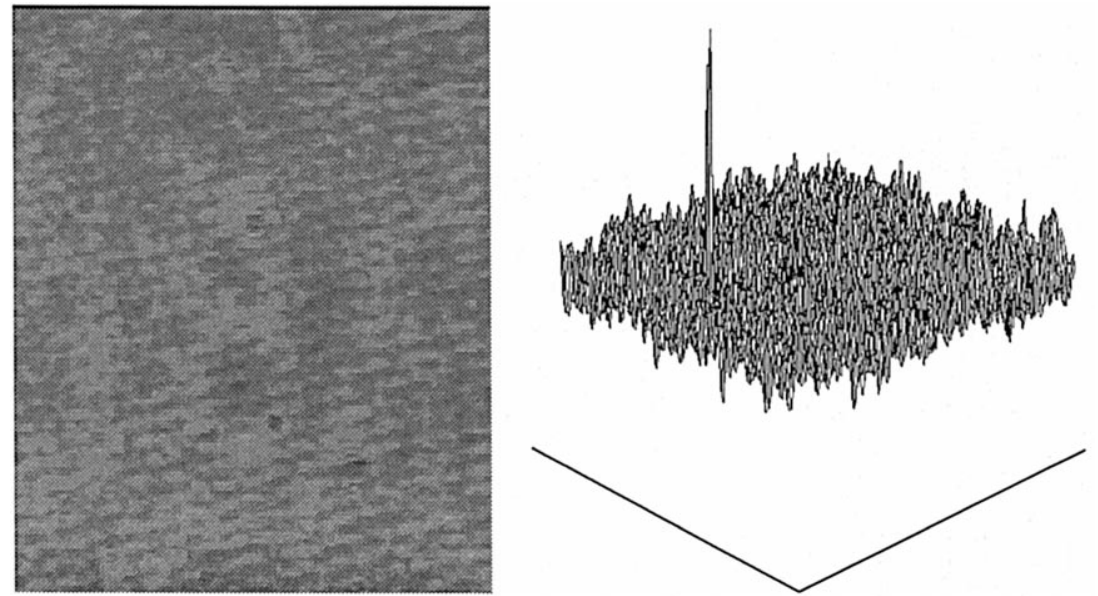

Fig. 5. Total correlation plane. Detection of the bottom satellite.

single modulator with a configuration with the required two values of $I_{\mathrm{BS}}$. This can be easily achieved with a phase-only response, which has a constant transmittance and a wide range of phase variation.

\section{Experimental procedure and results}

The scene chosen for the experimental demonstration is shown in Fig. 2, and the verification of the method consists in the detection of each satellite. In the optical setup, we have used a single LCD removed from an Epson EMP-3000 videoprojector. This panel has a VGA resolution $(640 \times 480$ pixels $)$ with a pixel pitch of $40 \times 40 \mu \mathrm{m}$. The whole process has been carried out by use of different configurations of a single modulator. As pointed out before, these panels can not reach $2 \pi$ radians of phase modulation owing at its low birefringence.

To display the required distribution of Eq. (7), we have chosen a $\mathrm{HC}$ configuration (Fig. 3) that gives a
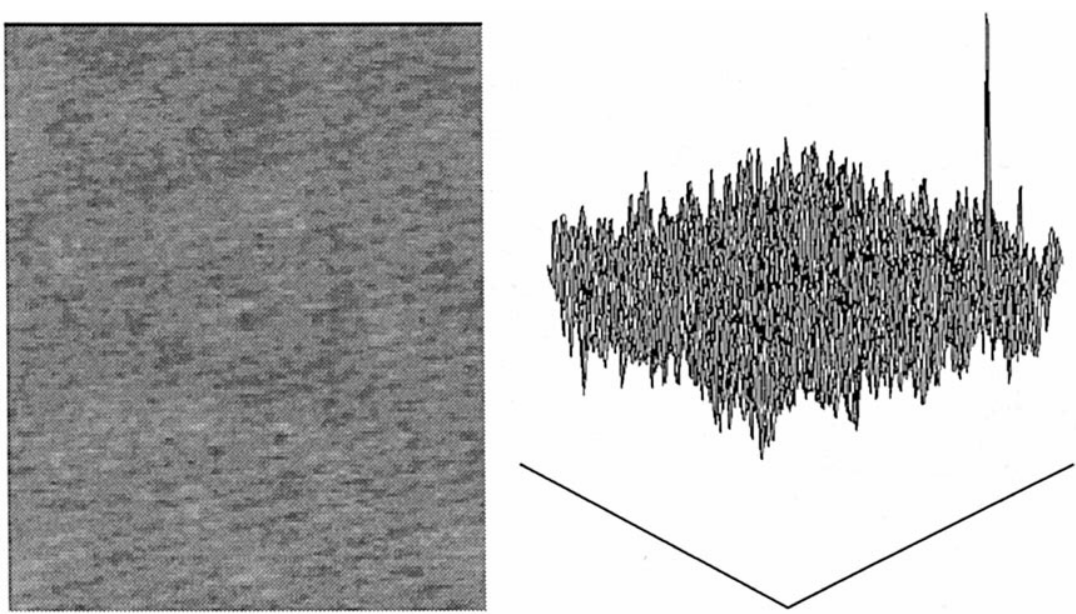

Fig. 6. Total correlation plane. Detection of the upper right satellite. 

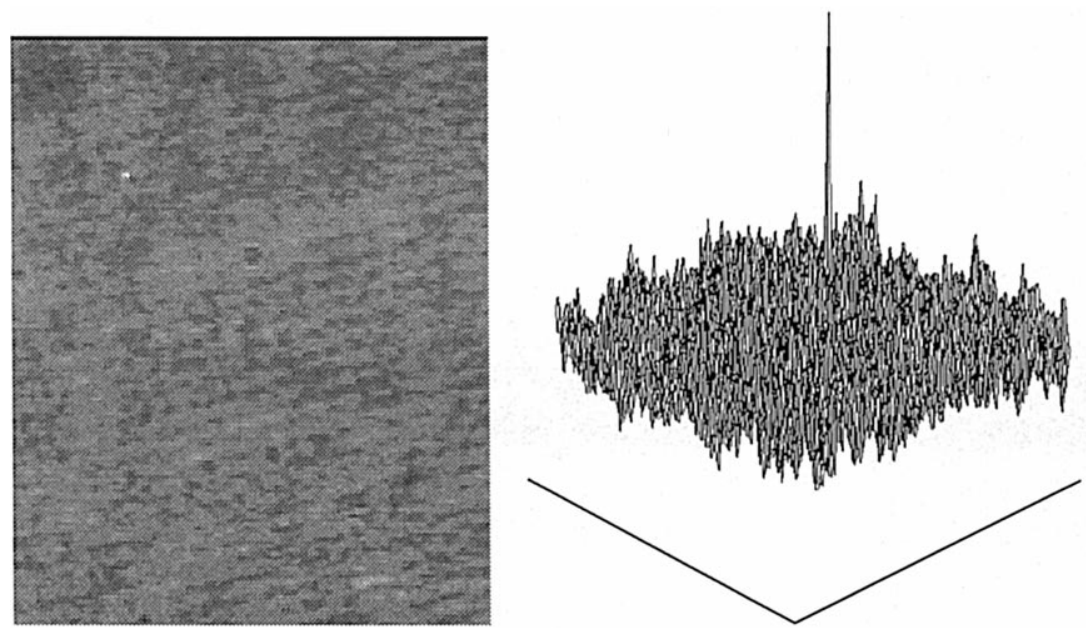

Fig. 7. Total correlation plane. Detection of the upper left satellite.

high contrast between the grey level $\mathrm{gl}=255$ and $\mathrm{gl}=0$, and a phase shift between $\mathrm{gl}=255$ and $\mathrm{gl}$ $=119$ of $\pi / 2$, as needed.

The sine distribution $I_{\mathrm{S}}$ (Eq. (5)) has been obtained by displaying the reference binary image with $\mathrm{gl}=255$ and $\mathrm{gl}=0$, and the binary scene image with $\mathrm{gl}=119$ and $\mathrm{gl}=0$. To obtain the threshold function $\left(I_{\mathrm{T}}\right)$ we have displayed separately the reference and the scene images with the aforementioned grey levels, respectively.
Once we have computed $I_{\mathrm{BS}}$ (Eq. (8)), we have to display it on the LCD with a configuration where two grey levels have the same transmittance and with relative phase shift of $\pi$. The operating curve that we have used is a $0-\pi$ phase-only modulation (Fig. 4) that was obtained with elliptically polarized light [13]. The two chosen grey levels we needed to display $I_{\mathrm{BS}}$ have been $\mathrm{gl}=0$ and $\mathrm{gl}=255$.

Displaying $I_{\mathrm{BS}}$, we can achieve the desired final correlation adding the suitable plane wave. Figs. 5-7
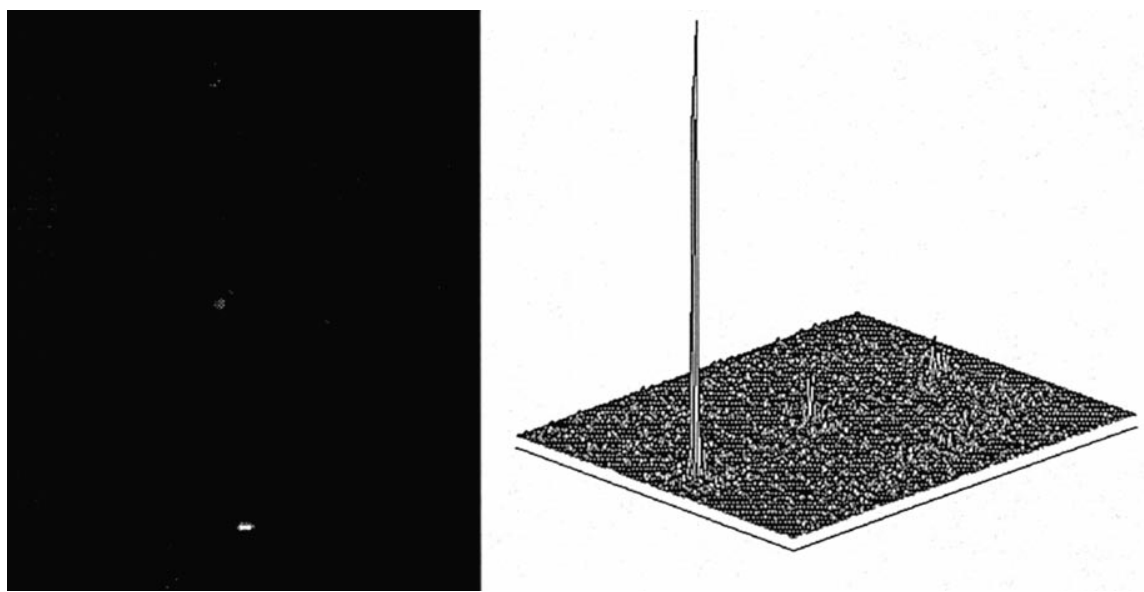

Fig. 8. Total correlation plane with background removed. Detection of the bottom satellite. 

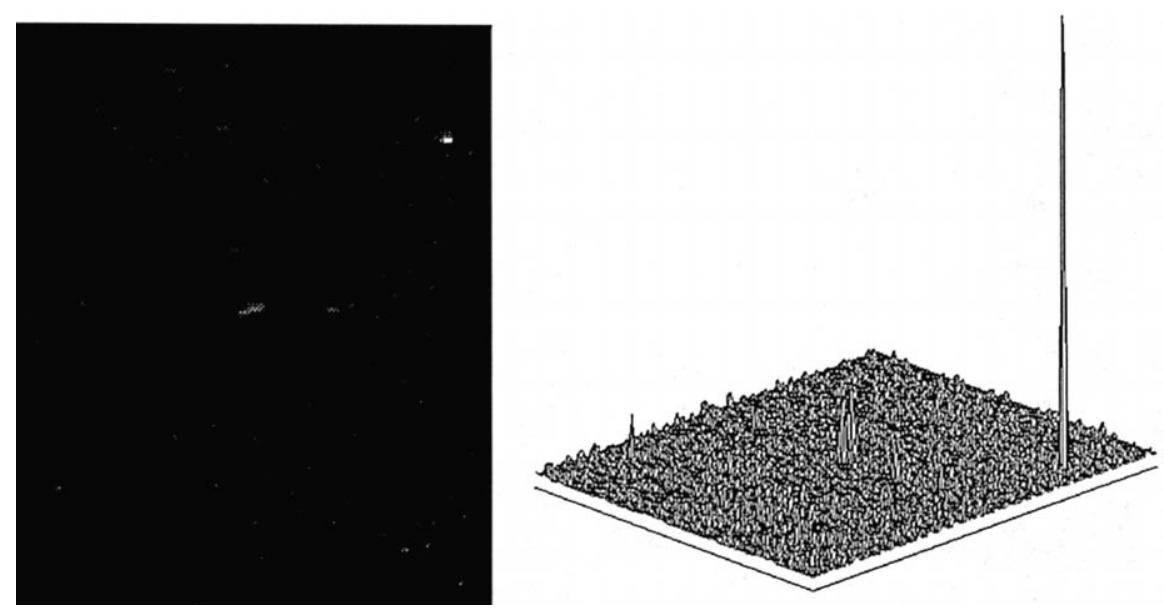

Fig. 9. Total correlation plane with background removed. Detection of the upper right satellite.

show the correlation planes when each satellite is detected. The detection peaks are higher than the background in the correlation planes, as shown in the 3D plots. Approximately, the background mean value is half the value of the detection peak. When we remove this background we can see the detection peaks clearer than before. Figs. 8-10 show the correlation results when it is removed.
The correlation results are similar than the ones we got when we used CGA panels [10]. Those devices could reach $2 \pi$ radians of phase modulation but there was a remaining transmittance that produced a non null central order. With those devices, the resolution was only $310 \times 242$ and inaccurate gray levels appeared due to an approximated pixel by pixel addressing of the modulators. With use of
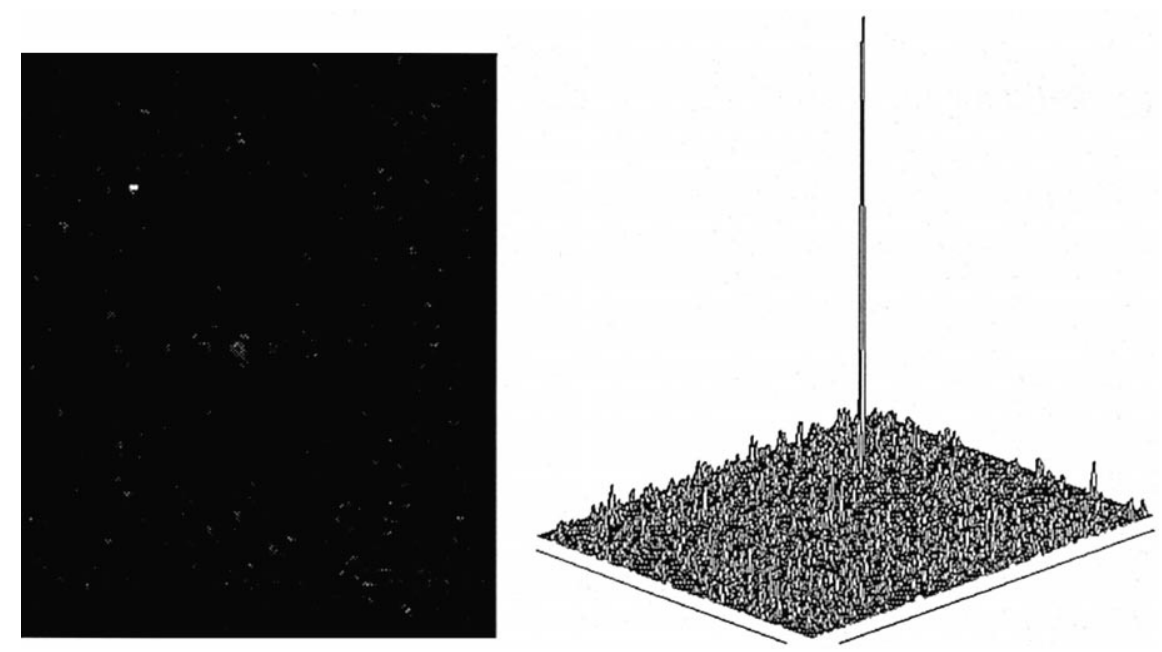

Fig. 10. Total correlation plane with background removed. Detection of the upper left satellite. 
VGA panels we have increased the resolution (640 $\times 480$ ), we have a pixel by pixel addressing of the LCDs and the needed operating curves are less restrictive than before. Another issue to take into account is that with this new method only three power spectra are required to compute the binary JPS while we needed five spectra to obtain the four-level JPS.

\section{Conclusions}

We have proposed a variation in our previous method to obtain a single centered correlation term. This new approach allows the use of displaying panels with flexible configurations because only two grey levels are needed. With this modification we can use modulators with less than $2 \pi$ phase modulation. However, the modified method requires an optical setup based on an interferometric architecture, involving an accurate experimental adjustment. Comparing this method with the previous one, we have reduced the number of power spectra required to obtain the computed JPS from five to three and we only need two gray levels to display it instead of four. Concerning the LCDs used, we have increased the devices resolution and now we have a real pixel by pixel addressing of the modulators. We have carried out an experimental verification of this approach with the interferometric setup achieving good detection results.

\section{Acknowledgements}

This paper has been supported in part by the CICYT (Comisión Interministerial de Ciencia y Tecnología) under project TAP97-0454.

\section{References}

[1] C.S. Weaver, J.W. Goodman, Appl. Opt. 5 (1966) 1248.

[2] J.E. Rau, J. Opt. Soc. Am. 56 (1966) 1490.

[3] F.T.S. Yu, X.J. Lu, Opt. Commun 52 (1984) 10.

[4] B. Javidi, C.J. Kuo, Appl. Opt. 27 (1988) 663.

[5] Q. Tang, B. Javidi, Appl. Opt. 32 (1993) 1911

[6] B. Javidi, Q. Tang, G. Zhang, Appl. Opt. 33 (1994) 6219.

[7] G. Lu, Z. Zhang, S. Wu, F.T.S. Yu, Appl. Opt. 36 (1997) 470.

[8] E. Martín-Badosa, A. Carnicer, I. Juvells, S. Vallmitjana, Meas. Sci. Technol. 8 (1997) 764.

[9] C.T. Li, S. Yin, F.T.S. Yu, Opt. Eng. 37 (1998) 58.

[10] I. Labastida, A. Carnicer, E. Martín-Badosa, I. Juvells, S. Vallmitjana, Appl. Opt. 38 (1999) 6111.

[11] B. Javidi, J. Wang, Q. Tang, Appl. Opt. 30 (1991) 4234

[12] B. Javidi, Appl. Opt. 28 (1989) 2358.

[13] J.L. Pezzaniti, R.A. Chipman, Opt. Lett. 18 (1993) 1567. 